\title{
'Lion Leadership Lessons Video Series' - Delivering Engineering Leadership Lessons to a Broad Audience
}

\section{Dr. Dean H. Lang, Pennsylvania State University, University Park}

Dr. Lang is currently the Associate Director of the Engineering Leadership Research Program at Penn State University. She holds a BS in Mechanical Engineering from West Virginia University, an MBA from Johns Hopkins University, and a PhD in Kinesiology with a focus on Biomechanics from Penn State University. Dr. Lang's previous professional experiences and research interests range from mechanical engineering facilities design to research that applied engineering and molecular biology approaches to the study of the skeletal response to mechanical loading. As a Mechanical Engineer, she worked on facility design projects involving mechanical systems that included heating, ventilation, air conditioning, and energy conservation systems, as well as R\&D of air conditioning equipment for Navy ships. Additional research interests have included the investigation of relationships among components of the indoor environment, occupants, and energy usage. Specifically, the effects of the indoor environment on occupant health and well-being and in parallel, how socially-mediated energy-saving strategies can increase awareness of energy use and/or increase energy saving behaviors. Dr. Lang's current research interests focus on identifying, assessing, and developing key skills, knowledge, attitudes, and other intrinsic and extrinsic factors required for engineers to effectively lead others, particularly other engineers and across cultures.

\section{Mr. Andrew Michael Erdman, The Pennsylvania State University}

Andrew M. "Mike" Erdman received his B.S. in Engineering Science from Penn State and his M.S. from USC. At Rocketdyne (Pratt \& Whitney), he helped design the Space Shuttle. As manager of Reactor Safety Analysis, Experimental Engineering, and Fluid Dynamics Technology at KAPL (Lockheed Martin), he conducted research for Naval Reactors. He currently serves as the Walter L. Robb director of Engineering Leadership and as an instructor in Engineering Science at Penn State. Erdman has chaired the local Jaycees, Department of Social Services Advisory Council, GE Share Board, and Curling Club; and served on the Human Services Planning Council, United Way, Chamber of Commerce, and Capital Fund Drive Boards of Directors. Erdman has also lectured on leadership topics at Penn State and RPI. He has served on the Penn State College of Engineering Advisory Council, helped establish an Alumni Advisory Board, and currently serves as the President of the College of Engineering Alumni Society. Affiliations include the Penn State Alumni Association, Centre County Chapter Board of Directors, President's Club, Nittany Lion Club, ASEE, ASME, AIAA, AKC, GRCA. He has been honored with a LMC/KAPL Leadership Award, GE Phillippe Award, PSEAS Outstanding service award, Jaycee International Senatorship, and an ESM Centennial Fellowship.

\section{Mrs. Meg Handley, Pennsylvania State University, University Park}

Meg Handley is currently the Associate Director for Engineering Leadership Outreach at Penn State University. Previously, Meg served as the Director of the Career \& Corporate Connection's office at the Smeal College of Business at Penn State University. Meg is a PhD candidate in Workforce Education at Penn State, where she is focusing on interpersonal behaviors and their impact on engineering leadership potential.

Meg is a board certified coach with experience in developing students' leadership and professional competencies through teaching and one-on-one coaching. She is most interested in developing student knowledge of leadership to impact their successful transition to the workplace. 


\title{
'Lion Leadership Lessons Video Series' \\ Delivering Engineering Leadership Fundamentals to a Broad Audience
}

\begin{abstract}
Leadership skills are in ever-growing demand among companies who recruit engineering graduates. This need has been recognized in numerous studies and addressed in engineering accreditation standards ${ }^{1-9}$. Many universities struggle to find space in a curriculum under constant pressure to cover broadening technical fields. Often these leadership courses are offered as options on top of existing requirements, resulting in students taking additional credits, at significant cost to them.
\end{abstract}

The Engineering Leadership Development (ELD) Program at Penn State University has offered an 18 credit minor in Engineering Leadership Development for over 20 years, with over 600 graduates. While approximately two times that many students have benefitted by taking courses in engineering leadership during that period, it still represents a fraction of the more than 20,000 who have graduated from the College of Engineering in the same time span. Further, anecdotal evidence has shown that, while many courses provide opportunities for students to work on or lead teams, without guidance on leadership skill development, they often struggle and do not reach their potential as leaders.

To extend the reach of engineering leadership skill development, Penn State's ELD program has developed a video series to be used in first year seminars to deliver leadership lessons to all freshmen engineering students. These video vignettes are meant to provide some basic leadership insights that students can use in their course work, on teams, in extra-curricular activities, personal interactions and management, and can carry through to their work after graduation. They are based on research into personal and team performance, and build upon the fundamental concepts of honesty, trust, fairness, respect, courage and accepting responsibility in all interpersonal interactions ${ }^{10}$.

The situations and roles were written with the help of and played by students and are intended to be both informative and entertaining. Each video depicts a situation that students are very likely to encounter, and includes a typical response, as well as a more productive approach. At the end of each video, we summarize key points to remember when students encounter similar situations as well as suggested follow-up activities that students could do independently or instructors could incorporate within their classroom instruction.

The videos were piloted for the first time during the Fall 2015 semester with a limited number of users, and brought into wider use in the Spring 2016, both by encouraging widerspread use within Penn State but also by offering the site to other university engineering leadership. At the end of the spring semester we will seek feedback from instructors as well as students. Students will be asked to provide feedback to assess how effective the videos were at developing an awareness and understanding of effective personal and team performance approaches when faced with situations that provide an opportunity to demonstrate engineering 
leadership competencies. Results from the feedback will be factored into revisions to the films, and included in a subsequent study.

\section{Introduction}

Storytelling is used across industries to help convey concepts about leadership and teamwork ${ }^{11}$. Developmental bibliotherapy (guided reading) is a tool that uses fictional written stories to help develop social, emotional, or psychological growth at all levels of development ${ }^{12-13}$. In 1949, Shrodes identified four stages of developmental bibliotherapy: 1) identification - where the reader identifies with a character in a story; 2) catharsis - when a reader is able to experience the emotions of the character of the story; 3 ) insight - a deeper understanding which is achieved through reflection on the identification that the reader makes with the characters and situations of the story; and 4) universalization - when a reader is able to apply the insights the reader has gained through reflection to situations they encounter in their own life ${ }^{14}$. Hebert and Spiers Neumeister (2001) extended these concepts to guided viewing of film ${ }^{15}$. Herbert suggests that film may enable individuals to appreciate the humorous aspects of their own situations while providing alternative solutions to similar problems they are experiencing.

Using the concepts from bibliotherapy applied to film, the authors used videos to develop story lines in an effort to build leadership knowledge in alignment with current student educational experiences within the context of engineering education. Although much of the literature is on the use of popular movies and films applied to therapeutic environments such as the social, emotional, or psychological growth of gifted adolescents ${ }^{14}$, the authors believe by developing story lines utilizing four main characters (Emily, Ashley, James, and Mike) within 13 separate video vignettes, the audience will get to know the characters and become interested in the challenges they face within each video.

In order to impact learning through identification, emotions, deeper understanding and universalization, the authors developed the characters whose actions represent documented/researched personality measures associated with Keirsey Temperaments (SJguardian, NF-idealist, NT-rational, and SP-artisan) $)^{16}$ and Meyers \& Briggs MBTI personality types (ESTJ, INFP, INTJ, and ESTP) ${ }^{17}$. This strategy paired with typical experiences faced by undergraduates, which require leadership characteristics, may help student learners with identifying and connecting with the emotions of the story, developing a deeper understanding of the concepts.

The four main characters represented the four temperaments from Kiersey and the Meyers Briggs personality types to the extent that the video scripts allowed the personalities being portrayed to align with those preferences: Emily - SJ (guardian), likely ESTJ personality type, Ashley - NF (idealist), likely INFP, James NT (rational), likely INTJ; and Mike SP (artisan), likely ESTP. At times, as in actual situations, the characters strayed from these temperaments to fulfill their roles.

The videos portray the main characters confronting typical experiences that undergraduate students' encounter, providing an opportunity for students to identify with the characters in an effort to build empathy and insight. Within each video, follow up activities are suggested to 
motivate the student to apply the insights gained through the videos. Throughout the video series each of the main characters develop and grow as leaders and in the culminating video celebrate a group win with financial backing from corporate investors.

\section{Approach to Development}

The focus of the films was to provide basic leadership lessons for freshmen entering the College of Engineering, specifically skills that would be valuable in their early engineering activities, such as teams, self-motivation, and accepting and giving feedback.

Guiding principles in developing the lessons and videos included:

- The lessons need to be aimed at basic leadership skills, those that would be required by students to be successful in foundational courses in engineering, and particularly when small teams are expected to work effectively together.

- The lessons need to be based on solid engineering leadership principles, as identified by research into the underlying tenets of each leadership lesson, with references provided for those who wish to delve more deeply into the topic.

- The lessons need to be informative, yet capture the listener. The student needs to be able to put him/herself in the "shoes" of the actor.

- The lessons need to be light and entertaining. The lessons need to NOT come across as another lecture, but rather a fun set of suggestions.

- Where appropriate, the video should show a typical, knee-jerk reaction to a situation, followed by a more constructive approach. The video should complete the "lesson" with key points to remember when faced with such a situation, and actions the student can take to improve their behavior.

- The lessons need to be concise with key learning points easily remembered and incorporated in future behaviors. The typical length of any one lesson should be kept to 4 to 6 minutes to maintain the students' attention.

- Hand-outs and easily retrieved electronic files with the key points should be provided to increase the retention and easy access for the students.

- Follow-up discussion by the instructor or activities for the students can help to make the lessons memorable. The suggested follow-up activities were developed to be easily incorporated into class assignments at the discretion of the instructor.

The scripts and the use of student actors are aimed at the freshmen, not the professional, and need to be viewed with that filter in place. The scripts were developed with significant input from an undergraduate teaching assistant in collaboration with the faculty of the engineering leadership program. Students from within and outside of the College of Engineering were used as actors. Student actors were purposely used to fill the roles of older characters such as professors, advisors, employees, managers, and venture capital representatives to add an additional element of humor. A local production company, heavily staffed with student interns, was engaged to do the recording and editing. An overriding consideration was that the films were not to be viewed as "professional" productions, but rather as a student effort. It is hoped that this will help provide a connection with the target audience. 


\section{Scope of the Lessons}

The lessons were developed to fit into a 15 week semester where the first and last of the sessions were dedicated to start-up and wrap-up of the class, resulting in 13 opportunities for leadership lessons. The following topics, along with the key take-aways and suggested followup actions were chosen for each segment:

\#1 Leadership Basics - an introduction to the objectives of and approach for the series of leadership lessons. Two completely opposite approaches to leading a team are played out - one where the appointed team leader demotivates the team, the other where the leader encourages team participants. The close-out asks the question "What type of leader would you want to follow?" and invites the audience to come along through the video series to learn about leadership and how to develop their own leadership skills. $\quad-5: 44$ minutes

\#2 Communication - how to improve your verbal communications, a key skill of leaders. The video begins with a student struggling to prepare for a presentation. He is mentored by a more practiced, confident speaker. Key take-aways include remembering to look at the audience, smile, project your voice, display confidence, and good posture ${ }^{18-20}$. Negatives to avoid include reading from notes, shifting stance, and fillers such as "um". The follow-up activity is to use a smartphone to record a practice session to identify areas to improve. $\quad-4: 44$ minutes

\#3 Cultural Sensitivity - how to avoid stereotyping and work well in culturally diverse teams. Students are seen sharing international foods when they encounter some cultural insensitivity. The interaction is followed by interviews with students from several different cultures, where differences are discussed and sensitivity to these differences is considered. The follow-up activity suggested is to interview an international student to learn more about their culture and some suggested interview topics are presented ${ }^{21-22}$.

$-11: 15$ minutes

\#4 Time Management - how to improve your personal organization to get more done in a day. A student volunteers to help organize a friend who is struggling with personal time management and setting priorities. Take-aways include understanding of the 4 quadrant Time Management Matrix $^{23}$ (Urgent, Not Urgent, Important, Not Important). Follow-up activities include logging actual activities for several days to understand where valuable time is lost. $-5: 49$ minutes

\#5 Constructive Relationships - building networks at school that can help expand horizons. As a class assignment a student interviews a laboratory owner which results in future opportunities to work as a lab assistant, travel, and receive a professional reference. The benefits $^{24}$ of this extended network are summarized at the end of the vignette, as well as recommendations to help reflect on how current contacts may be approached to expand their network. $-4: 19$ minutes

\#6 Working in Teams - overcoming some common pitfalls in teamwork. A new team begins to organize itself, and storming quickly becomes evident. As the team works through the disagreements, some actions are taken to help normalize their efforts. Tuckman's model ${ }^{25}$ is augmented with symptoms of and suggested actions for each stage of the team's development ${ }^{26-}$ 
27. The follow-up activity asks students to identify a group encounter where one team member is impacting the group and use that opportunity to ask questions of each team member to give them a chance to be heard to help reduce conflict. Students are then asked to reflect on the encounter. $-6: 12$ minutes

\#7 Feedback - giving and receiving constructive feedback. A team member who is chronically late to meetings arrives unprepared and is verbally attacked by the team leader for his latest misstep. The leader reconsiders her reproach and works to resolve the situation by giving constructive feedback. Take-aways include suggestions on giving effective feedback, including being timely, how to keep the discussion constructive, and minimizing defensive reactions. Suggestions on receiving feedback are also provided to encourage introspection ${ }^{28-30}$. The followup activity is to use the feedback framework to give a friend or group member constructive feedback followed by a reflection paper on what occurred, what worked, and what could have been done better. $-4: 55$ minutes

\#8 Negotiating - how to effectively sell your position and maintain positive relationships. An initial interaction results in a "win-lose" situation where a student imposes her position at the expense of cooperation from her teammate. A better team interaction is demonstrated, where ideas are accepted and worked collaboratively within the team. Prerequisites to achieving a "win-win" solution as well as steps in the negotiation are provided as the video ends ${ }^{23,31}$. Students are asked to use the negotiating win-win framework in a conflict or negotiation and to write a reflection on how the encounter went, including what worked and what didn't work. $-3: 33$ minutes

\#9 Motivating Self - suggestions on how to motivate yourself to perform at a high level when feeling time pressure. A well-organized, motivated student volunteers to help a student feeling pressure as the semester heads toward finals week. Recommendations are provided such as breaking assignments into smaller goals, rewarding yourself for achievements, and establishing a positive outlook ${ }^{31-33}$. This topic was specifically placed two thirds of the way through the video series to align with the most stressful part of the semester. Students are encouraged to apply the motivational tips and to use the available resources to help reduce stress and plan for the end of the semester and finals week.

$-4: 52$ minutes

\#10 Delegating - how to effectively share the work load in team situations. A team leader finds herself swamped with work as she accepts more than her share of responsibility. Based on suggested actions to more effectively delegate responsibilities, the leader more evenly distributes the workload. Suggested actions include clarifying deliverables and deadlines, recognizing team member skills, and establishing good feedback channels ${ }^{23,34-35}$. $\quad-3: 30$ minutes

\#11 Leading Meetings - how to establish productive meetings and keep them on track. A typical team meeting starts up, with some students arriving late, distractions with side conversations, and the leader not focused on the business at hand. Suggestions for improving meetings which result in a more productive future meeting, include establishing an agenda with timelines, starting on time, and staying on topic ${ }^{36}$. The suggested follow-up activity is to have team members take turns as the meeting leader during the remaining semester group meetings to practice the suggested tips for leading meetings.

$-3: 54$ minutes 
\#12 Leading Change - keys to encouraging acceptance of new ideas. A student on an internship assignment is faced with what he recognizes as a dated, inefficient process embedded in his employer's workplace. His professor suggests some proven techniques to recommend changes in the work processes and gain acceptance, including creating and communicating a new vision, establishing a sense of urgency, and celebrating wins ${ }^{37}$. Students are asked to identify a process or tool at work, school, or home that is outdated and use the suggested steps to outline a strategy for leading change. Students should reflect on each step and discuss what they think will be effective as well as any challenges they encountered.

$-5: 36$ minutes

\#13 Promoting Ideas - effectively presenting your ideas, a wrap up which includes elements of effective communication, building confidence, and encouraging change. A timid student is faced with a need to sell a new product idea to potential investors. Given suggestions on making an effective pitch, the student's presentation is successful in landing venture capital. Keys to selling ideas include understanding customers, markets and your competitive advantage ${ }^{38}$. Students are asked to identify a new product idea or service and develop a pitch.

$-4: 56$ minutes

\section{Feedback}

The videos were completed in December, 2015, with a roll-out of the complete suite of videos planned for the spring semester, 2016. Feedback from the limited number of students and instructors who have used the videos to date has been uniformly positive.

In addition to having a larger number of users in-house in the spring, we have opened the videos to anyone on YouTube. A more rigorous review will be conducted toward the end of the spring semester, including hits and comments from YouTube, and using focus group discussions with instructors and students. If necessary, certain videos may be remade.

\section{Lessons Learned}

The budget for the video series was limited, which led us to work with a small, start-up production firm who used student help. The quality of some of the video and audio was not up to expectations, and the schedule was significantly delayed due to the need for rework and delays in production. A completely in-house production may have allowed for more control over the schedule, with likely a loss in quality, or, conversely, investment in a more experienced production firm could have produced a higher quality, but at a higher cost. As a first attempt at these films, the compromise position appears appropriate.

Some of the scripts could have been shorter, targeting student attention spans. Additionally, the key take-aways for each film could be reduced in number to improve retention. For the more serious implementation, structured practice sessions would provide for a deeper understanding and more likely retention.

The films were targeted for the freshmen seminar series which is delivered by each department within the College of Engineering, with the department instructor team coordinated 
by one individual. The roll-out was requested through this individual, however, the communications with each department's seminar providers could be improved, such as holding an information session for the instructors. Further, more seminars are delivered in the fall than in the spring, so the number of students who may be viewing the films in the spring, when the assessment will be conducted, is low compared to what can be engaged in the fall. A second assessment may be appropriate in the fall.

\section{Conclusions}

The Lion Leadership Lessons Video Series is available for use by any interested party at the YouTube linkage https://www.youtube.com/playlist?list=PL5FtUzdCyVRBmHnGq8wTBatBdtCVg_0q. We suggest that interested users establish expectations that the lessons are purposely delivered in an informal, light-hearted, yet realistic manner so that students can picture themselves in these situations. Further, a discussion following the vignette, either reviewing the learning points or exercising the recommended actions can help to increase understanding and retention.

Feedback from users is highly encouraged and appreciated, please send us your feedback at ENGRLeadership@engr.psu.edu. It is hoped that these lessons will help your young engineering students quickly garner basic leadership skills that are important to their success in their academic and early professional careers.

\section{References}

[1] ABET. (2011). Criteria for accrediting engineering programs: Effective for review during the 2012-2013 accreditation cycle. Retrieved from www.abet.org/DisplayTemplates/DocsHandbook.aspx?id=3143.

[2] American Society for Engineering Education. (1995). “Educating tomorrow's engineers.” ASEE Prism, 4(9), $11-15$.

[3] Connelly, J. D., \& Middleton, J. C. R. (1996). Personal and professional skills for engineers: one industry's perspective. Engineering Science \& Education Journal, 5(3), 139-144. Retrieved from http://digitallibrary.theiet.org/content/journals/10.1049/esej_19960309

[4] Cox, M., Cekic, O., Ahn, B., Zhu, J. (2012). Engineering Professionals’ Expectations of Undergraduate Engineering Students. Leadership and Management in Engineering, 12, 60-70.

[5] Farr, J., \& Brazil, D. (2009). Leadership Skills Development for Engineers. Engineering Management Journal, 21(1), 3. http://doi.org/10.1080/10429247.2009.11431792

[6] Hartmann, B., Stephens, C., Jahren, C. (2015). Surveying industry needs for leadership in entry-level engineering positions. American Society for Engineering Education.

[7] Male, S. a. (2005). Generic Engineering Competencies : A Review and Modelling Approach. Education Research and Perspectives, 37(1), 25-51.

[8] Nair, C. S., Patil, A., \& Mertova, P. (2009). Re-engineering graduate skills - a case study. European Journal of Engineering Education, 34(2), 131-139. http://doi.org/10.1080/03043790902829281

[9] Nguyen, D. Q. (1998). The Essential Skills and Attributes of an Engineer: A Comparative Study of Academics, Industry Personnel and Engineering Students. Business, 2(1), 65-76. Retrieved from 
http://www.eng.monash.edu.au/uicee/gjee/vol1no3/paper4.htm\nhttp://citeseerx.ist.psu.edu/viewdoc/downl oad?doi=10.1.1.124.1502\&rep=rep1\&type=pdf

[10] Fishman, T. Editor (1999). The Fundamental Values of Academic Integrity, International Center for Academic Integrity.

[11] Carley, M. (1999). Training goes to the movies, Training and Development, 53(7), 5.

[12] Halsted, J. W. (1994). Some of my best friends are books: Guiding gifted readers from pre-school to high school. Dayton, OH: Ohio Psychology Press.

[13] Lenkowsky, R. (1987). Bibliotherapy: A review and analysis of the literature. The Journal of Special Education, 21, 123-132.

[14] Shrodes, C. (1949) Bibliotherapy: A theoretical and clinical experimental study. Unpublished doctoral dissertation, University of California, Berkeley.

[15] Hebert, T. P., Speirs Neumeister, K. L. (2001) Guided Viewing of Film: A Strategy for Counseling Gifted Teenagers. Journal of Secondary Gifted Education, Vol. 12, Issue 4, $224-235$.

[16] Keirsey Temperaments, retrieved from: http://keirsey.com/4temps/overview temperaments.asp

[17] MBTI, retrieved from: http://www.myersbriggs.org/my-mbti-personality-type/mbti-basics/the-16-mbtitypes.htm

[18] Effective Presentations In Engineering and Science: Guidelines and Video Examples. http://www.engr.psu.edu/speaking/SPEECH.html

[19] Anderson, C. (June 2013). How to Give a Killer Presentation. Harvard Business Review. https://hbr.org/2013/06/how-to-give-a-killer-presentation.

[20] Melissa Marshall TED talk, Talk Nerdy to Me. http://www.ted.com/talks/melissa_marshall_talk_nerdy to_me.

[21] Kiss, Bow, or Shake Hands 2nd Ed. Morrison and Conaway 2006, Adams Media, MA.

[22] Beyond Culture, Hall, 1989, Anchor Books, NY.

[23] Covey, S. R. (2004). The 7 habits of highly effective people: Powerful Lessons in Personal Change. New York: Free Press.

[24] Building Good Work Relationships Making Work Enjoyable and Productive. http://www.mindtools.com/pages/article/good-relationships.htm

[25] Tuckman, Bruce W. (1965). “Developmental Sequence in Small Groups”, Psychological Bulletin, 63, $384-399$.

[26] Lencioni, P. (2002). The Five Dysfunctions of a Team: A Leadership Fable. San Francisco, CA: Jossey-Bass.

[27] This American Life Audio 370: Ruining It for the Rest of Us. Dec 19, 2008 (first 12 minutes). http://www.thisamericanlife.org/play full.php?play=370

[28] Giving Constructive Feedback. http://www.cabrillo.edu/services/jobs/pdfs/giving-feedback.pdf

[29] Priolo, D. (2012). How To Give Constructive Feedback in 6 Easy Steps. http://info.profilesinternational.com/profiles-employee-assessment-blog/bid/102602/How-To-GiveConstructive-Feedback-in-6-Easy-Steps

[30] Giving Feedback Keeping Team Member Performance High, and Well-Integrated. http://www.mindtools.com/pages/article/newTMM 98.htm

[31] Waitley, D. (1986). The Psychology of Winning. Penguin Publishing Group.

[32] Penn State Student Affairs, Stress Management Tips: http://studentaffairs.psu.edu/counseling/showyoucare/managestress.shtml

[33] Penn State Student Affairs, EDGE online workshops: http://edge.psu.edu/ 
[34] Dale Carnegie Training (2012). Leadership Tips: Effective Delegation Process. http://blog.dalecarnegie.com/leadership/leadership-tips-effective-delegation-process/

[35] Zwilling, M. (2013). How To Delegate More Effectively In Your Business. Forbes http://www.forbes.com/sites/martinzwilling/2013/10/02/how-to-delegate-more-effectively-in-yourbusiness/.

[36] Mind Tools. Running Effective Meetings Establishing an Objective and Sticking to It. http://www.mindtools.com/CommSkll/RunningMeetings.htm

[37] Kotter, J.P. Leading Change: Why Transformation Efforts Fail. Harvard Business Review. Jan. 2007. http://www.businessperform.com/change-management/resistance_to_change.html.

[38] Porter, J. (2012) 10 Questions to Answer Before Pitching Investors. Entrepreneur. http://www.entrepreneur.com/article/225155. 